\title{
Mapping tumour heterogeneity with pulsed 3D CEST MRI in non-enhancing glioma at $3 \mathrm{~T}$
}

\author{
Esther A. H. Warnert ${ }^{1}$ (1) - Tobias C. Wood ${ }^{2} \cdot$ Fatih Incekara $^{1,3}$. Gareth J. Barker ${ }^{2}$ - Arnaud J. P. Vincent ${ }^{3}$. \\ Joost Schouten ${ }^{3}$. Johan M. Kros ${ }^{4} \cdot$ Martin van den Bent $^{5} \cdot$ Marion Smits $^{1} \cdot$ Juan A. Hernandez Tamames ${ }^{1}$
}

Received: 14 August 2020 / Revised: 22 January 2021 / Accepted: 26 January 2021 / Published online: 19 February 2021

(c) The Author(s) 2021

\begin{abstract}
Objective Amide proton transfer (APT) weighted chemical exchange saturation transfer (CEST) imaging is increasingly used to investigate high-grade, enhancing brain tumours. Non-enhancing glioma is currently less studied, but shows heterogeneous pathophysiology with subtypes having equally poor prognosis as enhancing glioma. Here, we investigate the use of CEST MRI to best differentiate non-enhancing glioma from healthy tissue and image tumour heterogeneity.

Materials \& Methods A 3D pulsed CEST sequence was applied at 3 Tesla with whole tumour coverage and 31 off-resonance frequencies (+6 to $-6 \mathrm{ppm}$ ) in 18 patients with non-enhancing glioma. Magnetisation transfer ratio asymmetry (MTRasym) and Lorentzian difference (LD) maps at $3.5 \mathrm{ppm}$ were compared for differentiation of tumour versus normal appearing white matter. Heterogeneity was mapped by calculating volume percentages of the tumour showing hyperintense APT-weighted signal.

Results LDamide gave greater effect sizes than MTRasym to differentiate non-enhancing glioma from normal appearing white matter. On average, $17.9 \% \pm 13.3 \%$ (min-max: $2.4 \%-54.5 \%$ ) of the tumour volume showed hyperintense LDamide in non-enhancing glioma.

Conclusion This works illustrates the need for whole tumour coverage to investigate heterogeneity in increased APT-weighted CEST signal in non-enhancing glioma. Future work should investigate whether targeting hyperintense LDamide regions for biopsies improves diagnosis of non-enhancing glioma.
\end{abstract}

Keywords Non-enhancing glioma $\cdot \mathrm{CEST} \cdot \mathrm{APT} \cdot \mathrm{NOE}$

\section{Introduction}

Chemical exchange saturation transfer (CEST) is a technique to create magnetic resonance imaging (MRI) contrasts by selective targeting of labile protons in endogenous and

Esther A. H. Warnert

e.warnert@erasmusmc.nl

1 Department of Radiology and Nuclear Medicine, Erasmus MC, Rotterdam, NL, the Netherlands

2 Institute of Psychiatry, Psychology and Neuroscience, King's College London, London, UK

3 Department of Neurosurgery, Erasmus MC, Rotterdam, NL, the Netherlands

4 Department of Pathology, Erasmus MC, Rotterdam, NL, the Netherlands

5 Department of Neurology, Erasmus MC, Rotterdam, NL, the Netherlands mobile proteins. An emerging clinical application of CEST imaging is the assessment of glioma via amide proton transfer (APT) weighted CEST, which recently has shown promise for glioma grading $[1,2]$ and the differentiation between pseudoprogression and radiation necrosis [3].

Although patients with non-enhancing gliomas are often included in studies investigating APT-weighted CEST MRI, the main aim is often differentiation of glioma grade based on the classic histological classification of low to high-grade tumours, where high-grade tumours often show enhancement and form the majority of the studied population. The current body of literature indicates that lower grade gliomas are mostly isointense on amide proton transfer (APT)weighted imaging, with potentially some areas of hyperintensity and that high-grade gliomas, i.e. glioblastomas, show increased APT-weighted CEST signal [1, 4]. However, in non-enhancing glioma, APT-weighted CEST could have an important role as these tumours can become very large and, 
like glioblastomas, have spatially varying pathophysiology and underlying molecular signatures [5]. The latter is of particular importance in light of the recently updated molecular classification of tumours as published by the World Health Organisation [6]. Whereas non-enhancing glioma used to be classified based on histology only, now two molecular parameters (IDH mutation and $1 \mathrm{p} / 19 \mathrm{q}$ codeletion) are of interest that lead to three distinct classes of non-enhancing glioma [7]. These three classes differ widely in prognosis, ranging from a median overall survival of more than 10 years to just over 1 year, similar to enhancing glioblastoma (grade IV). Moreover, each of these classes warrants a different treatment regime, which stresses the need for accurate diagnosis.

Additionally, although the number of CEST research studies including multi-slice image acquisition schemes is increasing, previous investigations including non-enhancing glioma often use single-slice CEST acquisitions [4, 8, 9]. Because this excludes investigation of the whole tumour volume, care needs to be taken when interpreting the above results found about APT-weighted CEST signal in nonenhancing glioma. However, combining CEST preparations with a rapid 3D read-out has previously been shown to allow for the collection of multiple saturation offsets whilst covering the whole tumour volume in clinically feasible scan times $[10,11]$.

Here we use a 3D pulsed CEST sequence to explore APT-weighted signal in the whole tumour, specifically for non-enhancing glioma. We compare amide-weighted magnetisation transfer ratio asymmetry $\left(\mathrm{MTR}_{\text {asym }}\right)$ and Lorentzian difference (LD) contrasts, CEST metrics widely used in the current literature. The former is commonly applied and is valued for its relative simplicity as it, in its essence, only requires measurement of the CEST effect when applying a $B_{1}$ saturation pulse at two off resonance frequency shifts ( $3.5 \mathrm{ppm}$ and $-3.5 \mathrm{ppm}$ ) in addition to a separate acquisition of multiple off-sets for $\mathrm{B}_{0}$-correction. The latter requires covering multiple off-resonance frequencies via the acquisiton of a full Z-spectrum to do Lorentzian fitting of the CEST signal. This analysis allows for separate investigation of signal contributions from amide protons at $3.5 \mathrm{ppm}$ and nuclear Overhauser enhancement (NOE) at $-3.5 \mathrm{ppm}$. Note that in CEST experiments NOE contributes signal between -1 and $-5 \mathrm{ppm}$, where aliphatic protons in mobile macromolecules are saturated. Via relayed-NOE this saturation is transferred to amide protons within the same molecule which will exchange with the free water pool [12]. Recently, NOE-weighted CEST signal has been shown to be correlated with prognosis [13, 14] and grading [15] of high-grade glioma. Therefore, we additionally investigate whether NOEweighted CEST is of interest for non-enhancing glioma.

\section{Materials and methods}

All images were acquired on a 3 T MRI scanner equipped with a 32-channel head coil (Discovery MR750, General Electric, Chicago, USA). All experiments were conducted in compliance with the declaration of Helsinki and under approval of the institutional ethics committee of the Erasmus MC (Rotterdam, NL), which is one out of 18 accredited medical research ethics committees in the Netherlands. Image analysis and statistical analysis were done with inhouse written Matlab scripts (R2015b, The MathWorks, Natick, USA) and the freely available FMRIB Software Library (FSL 5.0.9, Oxford, UK).

\section{Patient information}

Eighteen patients with newly diagnosed presumed low-grade glioma were recruited between March 2017 and March 2019. Patients were recruited as part of the Imaging Genomics study and were scanned at maximum 7 days before surgical resection or biopsy. Tumour samples were obtained and histopathologically examined by neuropathologists. Molecular classification of the $1 \mathrm{p} / 19 \mathrm{q}$ co-deletion and IDH mutation status was performed as part of the diagnostic routine by molecular biologists with targeted Next-Generation Sequencing (NGS) panels using an Ion Torrent Personal Genome Machine or Ion S5XL (Thermo Fisher Scientific). Patient characteristics can be seen in Table 1 .

\section{Image acquisition}

CEST images were acquired with a 3D spoiled gradient echo with $\mathrm{TR}=35.4 \mathrm{~ms}, \mathrm{TE}=6.9 \mathrm{~ms}, 12$ slices, slice thickness $=5 \mathrm{~mm}$, in-plane voxel size $=1.85 \times 1.85 \mathrm{~mm}^{2}$, matrix $=128 \times 128$, acceleration factor $=4$, similar to the pulsed CEST sequence used by Jones et al. [10]. Spectralspatial excitation pulses were used to avoid fat artefacts in images near the water peak. [16] A Gaussian saturation pulse was played every TR with duration $20 \mathrm{~ms}$ (duty cycle 56.5\%). Two CEST series were acquired at saturation powers $\mathrm{B}_{1}=2.3$ and $4.0 \mu \mathrm{T}$, and each series consisting of 31 frequency off-sets $( \pm 6.0, \pm 5.5, \pm 5.0, \pm 4.5, \pm 4.0, \pm 3$. $75, \pm 3.5, \pm 3.25, \pm 3.0, \pm 2.5, \pm 2.0, \pm 1.5, \pm 1.0, \pm 0.75, \pm$ $0.5, \pm 0.25,0 \mathrm{ppm})$. Note that the saturation powers used are stated as the root mean square $B_{1}$ across the saturation pulse. Two images were acquired without saturation pulses to obtain the equilibrium magnetisation $\left(\mathrm{M}_{0}\right)$ for reference, bringing the total to 33 images acquired in $\sim 5 \mathrm{~min}$. $\mathrm{A}_{1}$ map was created by using a multi-slice $2 \mathrm{D}$ gradient echo sequence $\left(\mathrm{TE}=12.8 \mathrm{~ms}, \mathrm{TR}=17 \mathrm{~ms}\right.$, flip angle $\left.=10^{\circ}\right)$ with voxel size and number of slices $\left(1.85 \times 1.85 \times 5 \mathrm{~mm}^{3}\right.$, 
Tabel 1 Patient characteristics

\begin{tabular}{|c|c|c|c|c|c|c|c|c|}
\hline \multirow[t]{2}{*}{ Patient } & \multirow[t]{2}{*}{$\mathrm{M} / \mathrm{F}$} & \multirow[t]{2}{*}{ Age } & \multirow[t]{2}{*}{ Diagnosis (WHO 2016) } & \multirow[t]{2}{*}{ IDH mutation } & \multirow{2}{*}{$\begin{array}{l}1 \mathrm{p} / 19 \mathrm{q} \\
\text { codeletion }\end{array}$} & \multirow{2}{*}{$\begin{array}{l}\text { Tumour vol- } \\
\text { ume }(\mathrm{ml})\end{array}$} & \multicolumn{2}{|c|}{$\mathrm{LD}_{\text {amide }}(3.8 \mu \mathrm{T})$} \\
\hline & & & & & & & $\begin{array}{l}\text { Hyperintense } \\
\text { volume }(\mathrm{ml})\end{array}$ & $\begin{array}{l}\text { Hyperintense } \\
\text { volume (\%) }\end{array}$ \\
\hline P01 & M & 33 & Oligodendroglioma - grade II & Yes & Yes & 43.7 & 1.1 & 2.4 \\
\hline P02 & $\mathrm{F}$ & 57 & Oligodendroglioma - grade II & Yes & Yes & 177.6 & 41.2 & 23.2 \\
\hline P03 & $\mathrm{F}$ & 55 & Oligodendroglioma - grade II & Yes & Yes & 25.1 & 1.9 & 7.5 \\
\hline P04 & M & 35 & Oligodendroglioma - grade II & Yes & Yes & 227.1 & 57.0 & 25.1 \\
\hline P05 & M & 35 & Oligodendroglioma - grade II & Yes & Yes & 8.7 & 1.9 & 21.3 \\
\hline P06 & M & 31 & Oligodendroglioma - grade II & Yes & Yes & 16.9 & 1.1 & 6.4 \\
\hline P07 & M & 42 & Oligodendroglioma - grade II & Yes & Yes & 20.8 & 4.6 & 22.1 \\
\hline P08 & M & 39 & Astrocytoma - grade II & Yes & No & 80.0 & 28.1 & 35.1 \\
\hline P09 & $\mathrm{F}$ & 54 & Astrocytoma - grade III & Yes & No & 37.5 & 7.8 & 20.9 \\
\hline P10 & $\mathrm{F}$ & 46 & Astrocytoma - grade III & Yes & No & 62.6 & 21.0 & 33.6 \\
\hline P11 & M & 40 & Astrocytoma - grade III & Yes & No & 29.5 & 3.8 & 13.0 \\
\hline P12 & $\mathrm{F}$ & 24 & Astrocytoma - grade III & Yes & No & 79.7 & 4.1 & 5.2 \\
\hline P13 & M & 65 & Glioblastoma—grade IV & No & No & 12.8 & 7.0 & 54.5 \\
\hline P14 & M & 50 & Anaplastic astrocytoma - grade III & No & No & 13.8 & 1.8 & 13.4 \\
\hline P15 & M & 77 & Glioblastoma - grade IV & No & No & 20.2 & 1.2 & 5.8 \\
\hline P16 & M & 60 & Glioblastoma - grade IV & No & - & 40.5 & 5.4 & 13.3 \\
\hline P17 & M & 50 & Glioblastoma - grade IV & No & No & 78.6 & 9.1 & 11.5 \\
\hline P18 & M & 56 & Glioblastoma - grade IV & No & No & 13.5 & 1.1 & 8.0 \\
\hline
\end{tabular}

matrix $=128 \times 128 \times 12)$ matched to the CEST sequence. $\mathrm{T}_{1}$-weighted (3D IR FSPGR, TE $=2.1 \mathrm{~ms}, \mathrm{TR}=6.1 \mathrm{~ms}$, voxel size $=1 \times 1 \times 0.5 \mathrm{~mm}^{3}$, field of view $256 \mathrm{~mm}, 352$ slices) and $\mathrm{T}_{2}$-weighted FLAIR (3D spin echo read-out, voxel size $=1 \times 1 \times 1.6 \mathrm{~mm}^{3}$, matrix size $=224 \times 224 \times 264$, $\mathrm{TR} / \mathrm{TE} / \mathrm{TI}=6000 \mathrm{~ms} / 112.9 \mathrm{~ms} / 1893 \mathrm{~ms}$ ) structural images were additionally acquired. Note that the 3D CEST acquisition was planned to cover the whole tumour, as indicated by the $\mathrm{T}_{2}$-FLAIR hyperintense area.

\section{Image analysis}

Motion correction of the CEST image series was done by linear registration of each image within a series to the $6 \mathrm{ppm}$ image and a cost function based on mutual information ( $m c f$ lirt, within FSL v5.0.9, Oxford, UK), after which linear registration was used to register the CEST images to the magnitude of the $\mathrm{B}_{1}$-map. Z-spectra were calculated by dividing the images acquired with off-resonance saturation pulses by the average of the two $\mathrm{M}_{0}$ images. A Lorentzian curve was fitted to the Z-spectra using the data points with off-resonance frequency shifts of $\pm 6 \mathrm{ppm}$ and those from -1.75 to $1.75 \mathrm{ppm}$. This fit was first used for $\mathrm{B}_{0}$-correction, by shifting each spectrum by the frequency shift of the minimum value of the Lorentzian fit. Amide-weighted MTR $_{\text {asym }}$ was calculated according to the methods described by Zhou et al. [17], using the $\mathrm{B}_{0}$-corrected $\mathrm{Z}(3.5 \mathrm{ppm})$ and $\mathrm{Z}(-3.5 \mathrm{ppm})$. Lorentzian Difference (LD) analysis was used to obtain maps for $\mathrm{LD}_{\text {amide }}$ at $3.5 \mathrm{ppm}$ and $\mathrm{LD}_{\mathrm{NOE}}$ at $-3.5 \mathrm{ppm}$ [18-20]. The $\mathrm{MTR}_{\text {asym }}$ and LD contrasts were calculated for both $\mathrm{B}_{1}$ saturation powers acquired. Contrast-based $\mathrm{B}_{1}$ correction was then carried out according to previously described methods, including the use of an artificial $\mathrm{B}_{1}=0 \mu \mathrm{T}$ [21], resulting in voxel-wise $\mathrm{B}_{0}-\& \mathrm{~B}_{1}$-corrected $\mathrm{MTR}_{\mathrm{asym}}, \mathrm{LD}_{\text {amide }}$, and $\mathrm{LD}_{\mathrm{NOE}}$. To avoid extrapolation in voxels with a $\mathrm{B}_{1}$ below the nominal value of $\mathrm{B}_{1}=4.0 \mu \mathrm{T}$, in the remainder of the patient data the results are calculated for the images resulting from the $\mathrm{B}_{1}$-correction with $\mathrm{B}_{1}$ values of 2.5 and $3.8 \mu \mathrm{T}$.

Tumour regions of interest (ROI) were generated semiautomatically by delineating the hyperintense area on the $\mathrm{T}_{2}$-weighted FLAIR images using ITKSnap [22]. Note that no areas of necrosis were visually identifiable on the images acquired for the grade IV tumours $(N=6)$. The contralateral normal-appearing white matter (NAWM) ROI was generated by segmentation of white matter in the $\mathrm{T}_{1}$-weighted images (fast, within FSL v5.0.9, Oxford, UK) and a linear registration of this segmentation to the FLAIR image. NAWM was determined by using the white matter contralateral to the tumour in all slices that also included the tumour segmentation. Per patient, average tumour and NAWM MTR ${ }_{\text {asym }}$, $\mathrm{LD}_{\text {amide }}$, and $\mathrm{LD}_{\mathrm{NOE}}$ were calculated.

To determine the extent of hyperintense amide-weighted CEST signal within the tumour per contrast and per patient, for each patient a threshold was determined as follows: 
$S_{\text {thresh }}>\overline{S_{\text {amide }, N A W M}}+2 * \sigma_{\text {NAWM }}, S_{\text {thresh }}>\overline{S_{\text {amide }, N A W M}}+2 * \sigma_{\text {NAWM }}$,

where $\overline{S_{\text {amide }, N A W M} S_{\text {amide } N A W M}}$ is the average amideweighted signal in NAWM and $\sigma_{N A W M}$ is the standard deviation of $S_{\text {amide }}$ in the NAWM. The percentage of voxels surpassing this threshold within the tumour ROI, as determined by dividing the numbers of voxels surpassing $S_{\text {thresh }}$ within the tumour ROI by the total number of voxels covering the $\mathrm{T}_{2}$-weighted FLAIR hyperintense tumour area, was calculated per slice and for the whole tumour for each patient. $S$ represents the four different amideweighted contrasts calculated: $\mathrm{MTR}_{\text {asym }}$ and $\mathrm{LD}_{\text {amide }}$, both calculated for $\mathrm{B}_{1}$ is 2.5 and $3.8 \mu \mathrm{T}$.

\section{Statistical analysis}

Mixed effects linear regression models were fitted to determine whether there were significant effects of the fixed effects $R O I$ and $B_{1}$ on the CEST contrasts generated in the patient data $\left(\mathrm{MTR}_{\mathrm{asym}}, \mathrm{LD}_{\text {amide, }}\right.$ and $\left.\mathrm{LD}_{\mathrm{NOE}}\right)$. The $R O I$ factor contained two levels (NAWM and Tumour) and $B_{1}$ contained two levels $(2.5$ and $3.8 \mu \mathrm{T})$. To compare the different CEST contrasts for differentiating tumour tissue from NAWM effect sizes (Cohen's $d$ ) were calculated for all three contrasts.
A mixed effects linear regression model was also used to investigate whether there was a significant effect of contrast (two levels, $\mathrm{MTR}_{\text {asym }}$ and $\mathrm{LD}_{\text {amide }}$ ) or $B_{1}$ (two levels, 2.5 and $3.8 \mu \mathrm{T}$ ) on the volume percentage of the tumour showing hyperintense amide-weighted signal.

\section{Results}

Group averaged z-spectra, MTR $_{\text {asym }}$ and LD are plotted in Fig. 1. Group averaged values for all three CEST contrasts per ROI are stated in Table 2. For $\mathrm{MTR}_{\text {asym }}, \mathrm{LD}_{\text {amide }}$, and $\mathrm{LD}_{\mathrm{NOE}}$ the mixed-effects linear regression showed a significant effect of ROI ( $p<0.05$, Bonferroni corrected), indicating significant differences found between NAWM and tumour tissue. Post-hoc paired t-tests illustrate that only for $\mathrm{MTR}_{\text {asym }}$ and $\mathrm{LD}_{\text {amide }}$ there are significant increases in tumour tissue versus NAWM (Table 2). The largest effect size for differentiating the whole tumour ROI and NAWM was found for $\mathrm{LD}_{\text {amide }}$ when using a saturation power of 3.8 $\mu \mathrm{T}$ (Table 2).

Examples of the images generated by thresholding the $\mathrm{LD}_{\text {amide }} \mathrm{CEST}$ maps generated for $\mathrm{B}_{1}=3.8 \mu \mathrm{T}$ can be seen in Fig. 2. The hyperintense $\mathrm{LD}_{\text {amide }}$ voxels were heterogenously distributed across the tumours, as illustrated by Fig. 3. This figure illustrates that not necessarily all slices of the tumour
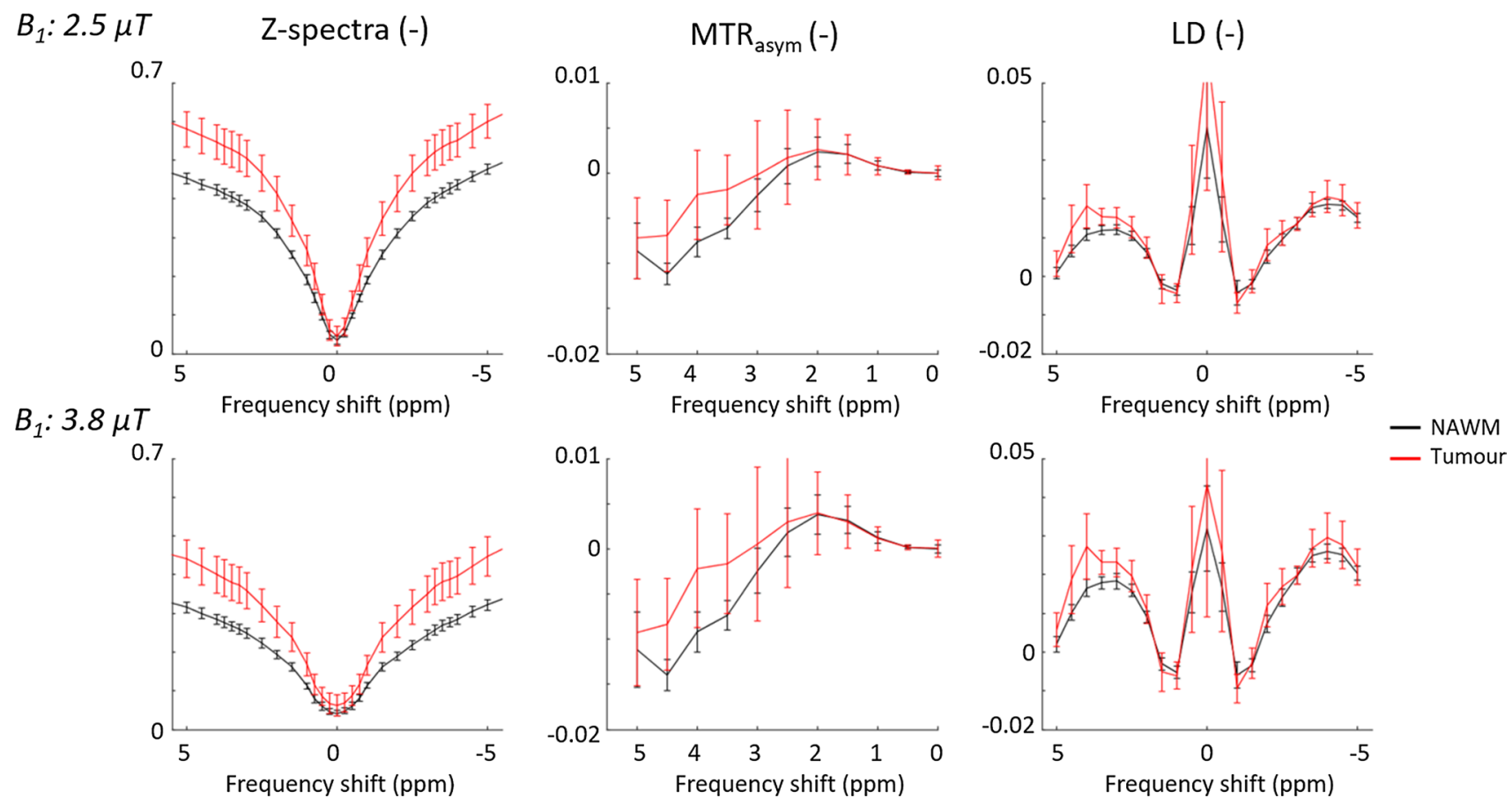

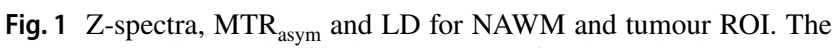
top row contains results for the $\mathrm{B}_{1}$ power of $2.5 \mu \mathrm{T}$, the bottom row contains results for the $\mathrm{B}_{1}$ power of $3.8 \mu \mathrm{T}$. Results are group averaged $(\mathrm{N}=18)$ and errorbars represent the standard deviation across the group. The Z-spectra in the left column are corrected for $\mathrm{B}_{0}$ inhomogeneity, the MTR $_{\text {asym }}$ and LD plots are additionally contrast $B_{1}$ -corrected 
Table 2 Averages and Cohen's d effect size for CEST contrasts in non-enhancing glioma patients $(\mathrm{N}=18)$

\begin{tabular}{lllll}
\hline & NAWM & Tumour & $\begin{array}{l}p \text {-value } \\
\text { Tumour vs NAWM }\end{array}$ & $\begin{array}{l}\text { Cohen's d } \\
\text { Tumour vs } \\
\text { NAWM }\end{array}$ \\
\hline$B_{I}=2.5 \mu T$ & $-0.006 \pm 0.001$ & $-0.002 \pm 0.004$ & $<0.001^{*}$ & 1.1 \\
$\mathrm{MTR}_{\text {asym }}$ & $0.012 \pm 0.001$ & $0.015 \pm 0.002$ & $<0.001^{*}$ & 1.6 \\
$\mathrm{LD}_{\text {amide }}$ & $0.017 \pm 0.002$ & $0.019 \pm 0.003$ & 0.200 & 0.3 \\
$\mathrm{LD}_{\mathrm{NOE}}$ & & & & 1.0 \\
$B_{1}=3.8 \mu T$ & $-0.007 \pm 0.002$ & $-0.002 \pm 0.006$ & $<0.001^{*}$ & 1.7 \\
$\mathrm{MTR}_{\text {asym }}$ & $0.018 \pm 0.002$ & $0.023 \pm 0.003$ & $<0.001^{*}$ & 0.4 \\
$\mathrm{LD}_{\text {amide }}$ & $0.025 \pm 0.002$ & $0.027 \pm 0.005$ & 0.083 & \\
$\mathrm{LD}_{\mathrm{NOE}}$ & & & & \\
\hline
\end{tabular}

* NAWM and tumour value significantly different, $p<0.05$, Bonferroni corrected

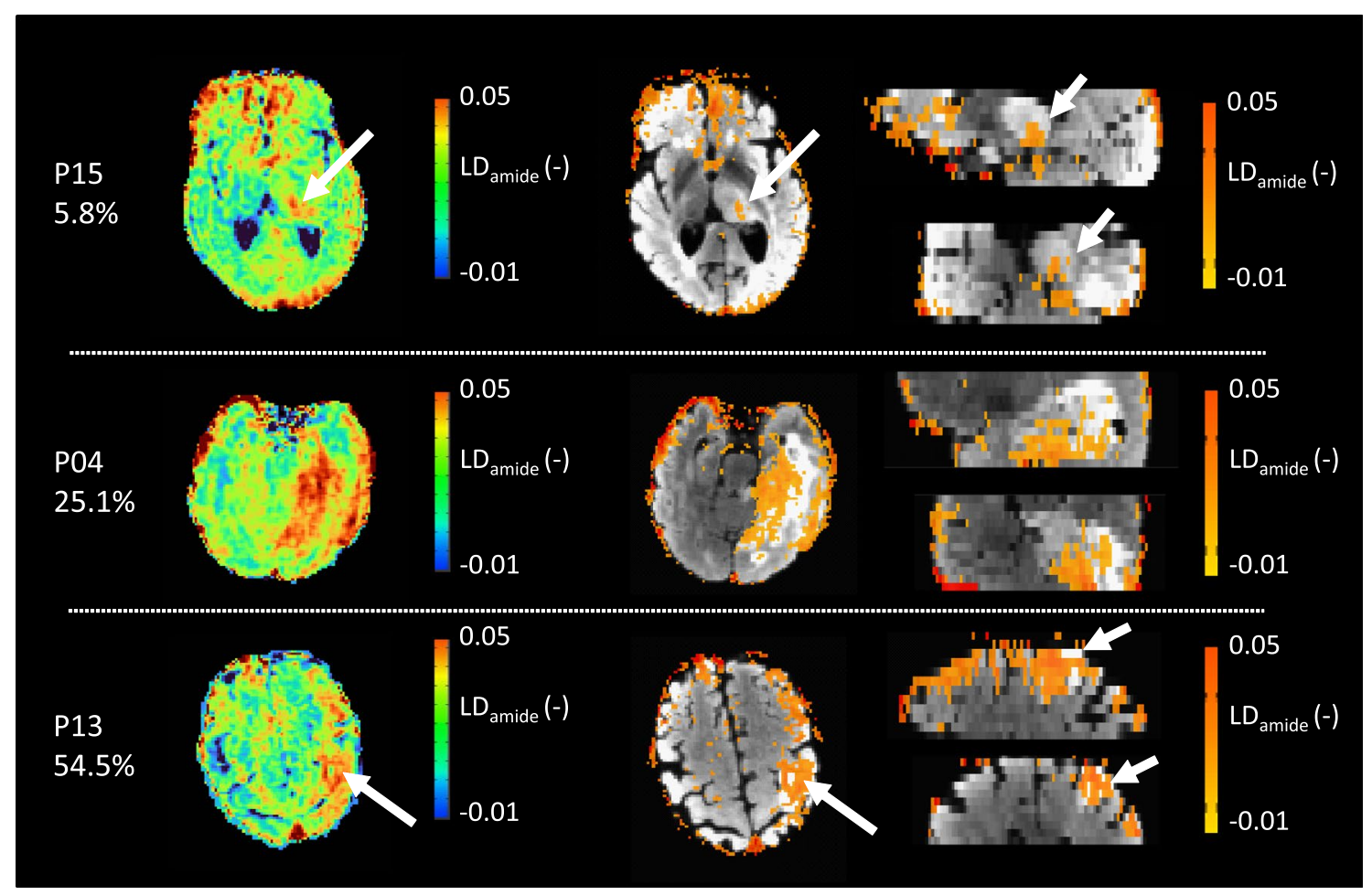

Fig. 2 Examples of $\mathrm{LD}_{\text {amide }}$ images acquired for three different patients with a $\mathrm{B}_{1}$ saturation power of $3.8 \mu \mathrm{T}$. Each of these patients has a different volume percentage of voxels with an increased $\mathrm{LD}_{\text {amide }}$, which is stated below the patient number. The left colum

contain hyperintense $\mathrm{LD}_{\text {amide }}$ signal. For example, in P01 the three most proximal slices of the tumour show no hyperintense $\mathrm{LD}_{\text {amide }}$ voxels. Figure 3 also illustrates that in 14 out of 18 patients the largest area of hyperintense $\mathrm{LD}_{\text {amide }}$ is not found in the slice with the largest tumour volume present. As an example, for P015 the slice with the largest amount of tumour voxels is slice 4, which contains no hyperintense $\mathrm{LD}_{\text {amide }}$ voxels. shows the unthresholded $\mathrm{LD}_{\text {amide }}$ maps, followed by the map thresholded based on the NAWM LD ${ }_{\text {amide }}$. The thresholded images are overlaid on the $\mathrm{T}_{2}$-weighted FLAIR and axial (left), sagittal (top), and coronal slices (bottom) are presented

On average, $17.9 \% \pm 13.3 \%$ of the tumour volume showed hyperintense $\mathrm{LD}_{\text {amide, }}$, which was based on a calculated $S_{\text {thresh }}$ of $0.031 \pm 0.003(N=18)$. The hyperintense tumour volume percentages found for $\mathrm{LD}_{\text {amide, } \mathrm{B} 1=2.5 \mu \mathrm{T}}, \mathrm{MTR}_{\mathrm{asym}, \mathrm{B} 1=2.5 \mu \mathrm{T}}$, and $\mathrm{MTR}_{\mathrm{asym}, \mathrm{B} 1=3.8 \mu \mathrm{T}}$ were $17.1 \% \pm 12.4 \%, 12.7 \% \pm 11.7 \%$, and $13.1 \pm 13.0 \%$, respectively (boxplots in Fig. 4). Although on average the $\mathrm{LD}_{\text {amide }}$ contrasts lead to a larger volume showing hyperintense signal in APT-weighted imaging, mixed-effects linear regressions did not show a significant effect of contrast 


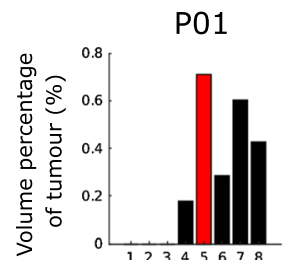

P07

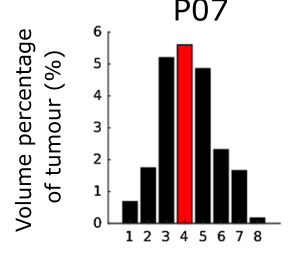

P13

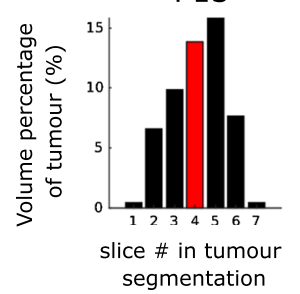

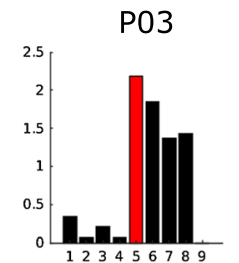

P09

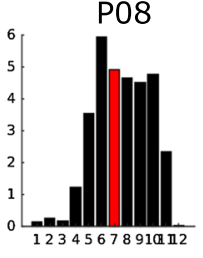

P14

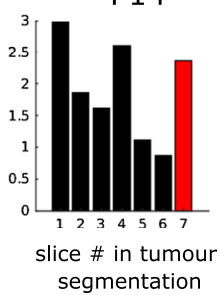

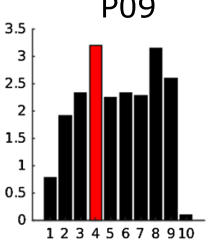

P15

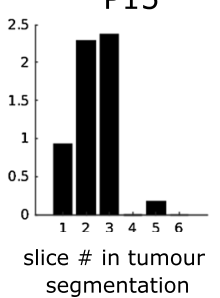

P04

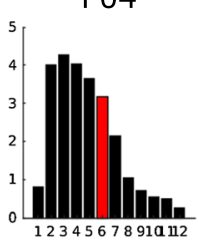

P10

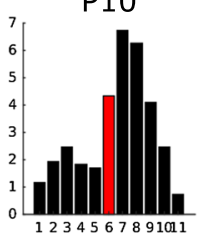

P16

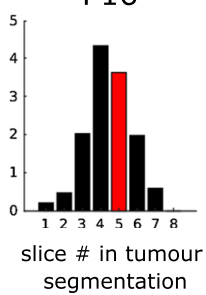

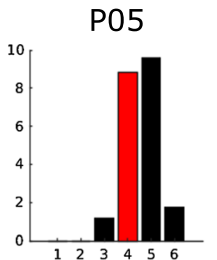

P11

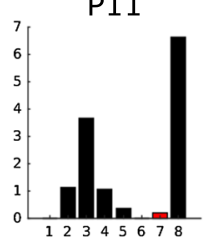

P17

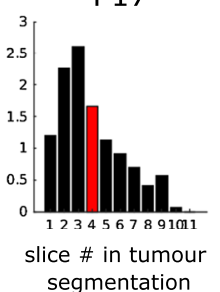

P06

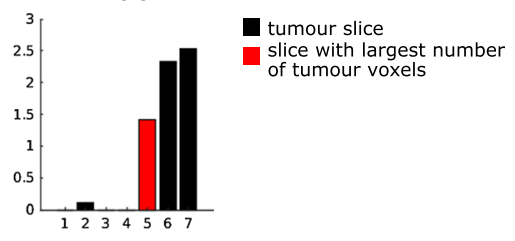

P12

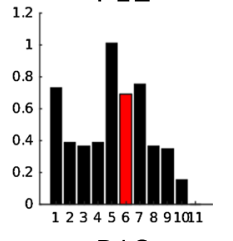

P18

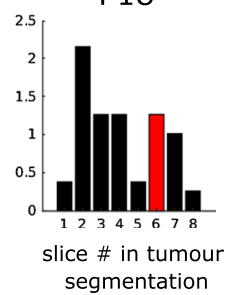

Fig. 3 Volume percentage of tumour showing hyperintense $\mathrm{LD}_{\text {amide }}$ for the CEST acquisition with a $\mathrm{B}_{1}$ saturation power of $3.8 \mu \mathrm{T}$. Each plot contains the volume percentages per slice containing tumour tissue for one patient. Slice \#1 is the most proximal slice. Note that each tumour has a different volume, reflected by different number of slices containing tumour tissue. The red bars indicate the slice with the largest number of tumour voxels. For P15 this is slice 4, which does not contain hyperintense $\mathrm{LD}_{\text {amide }}$ voxels

\section{Hyperintense APT-weighted tumour volume}

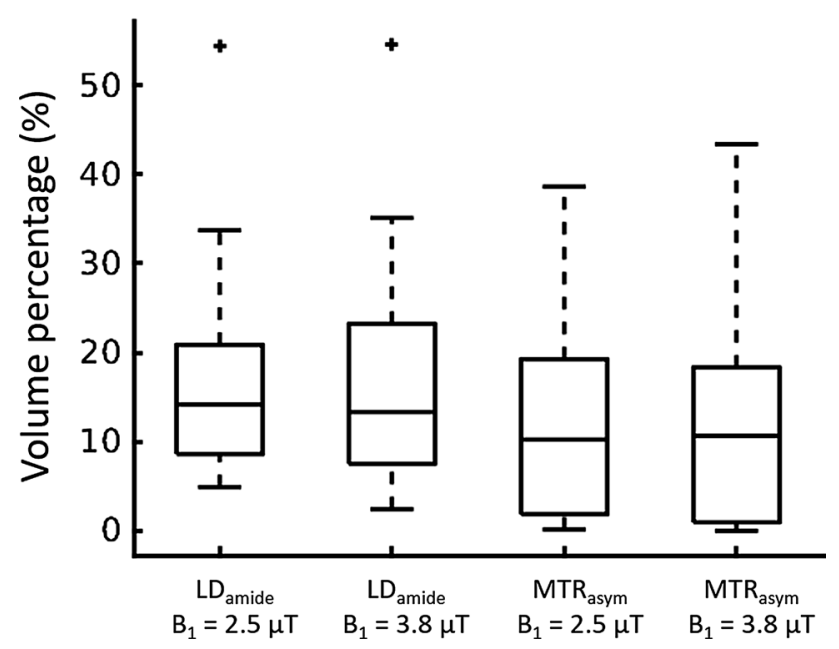

Fig. 4 Boxplots of volume percentages showing hyperintense APTweighted signal for $\mathrm{MTR}_{\text {asym }}$ and $\mathrm{LD}_{\text {amide }}$, for $\mathrm{B}_{1}=2.5 \mu \mathrm{T}$ and $3.8 \mu \mathrm{T}$ $(N=18)$. Although on average the $\mathrm{LD}_{\text {amide }}$ contrast leads to a slightly larger volume showing hyperintense signal in APT-weighted imaging, mixed-effect ANOVA did not show a significant effect of contrast on the volumes calculated $(p=0.045)$. Additionally, no significant effect of $\mathrm{B}_{1}$ power on the hyperintense volume percentage was found $(p=0.795)$ on the volumes calculated ( $p=0.045$, which is larger than the Bonferroni corrected p-value threshold). Additionally, no significant effect of $B_{1}$ power on the hyperintense volume percentage was found (mixed-effects linear regression, $p=0.795$ ).

\section{Discussion}

We investigated APT-weighted CEST MRI in non-enhancing glioma and showed that Lorentzian difference analyses are preferred in these type of tumours over the use of $\mathrm{MTR}_{\text {asym }}$ calculations since the largest effect size to differentiate tumour from NAWM was found for $\mathrm{LD}_{\text {amide }}$. Another important finding of this study is that, despite the majority of the non-enhancing glioma volume showing isointense signal amide-weighted CEST images, on average approximately $18 \%$ of the total volume of the $\mathrm{T}_{2}$-FLAIR hyperintense area showed hyperintensity on $\mathrm{LD}_{\text {amide }}$ images. The large area of isointense signal within these tumours is as expected, as earlier work in which non-enhancing glioma is included in patient populations has also found largely isointense APT-weighted CEST contrast [1, 4]. However, this work in which whole tumour coverage of non-enhancing glioma is done indicates that there are areas with hyperintense APT-weighted signal. This finding has two important implications: (1) it illustrates the spatial heterogeneity 
of pathophysiology within non-enhancing glioma and (2) stresses the need for covering the whole tumour when acquiring APT-weighted CEST images.

In particular in light of the spatial heterogeneity in pathophysiology and molecular signatures in non-enhancing glioma [5], the regions with hyperintense amide-weighted CEST signal may be areas where aggressive tumour tissue is present. This is an hypothesis strengthened by the potential sensitivity of APT-weighted CEST to protein build-up during cell proliferation, as for instance shown by Togao et al. [8] and Jiang et al.[23, 24] in a correlation between MTR $_{\text {asym }}$ and the Ki-67 labelling index, a histopathological marker of cell proliferation, in patients with low- and high-grade glioma. In a pre-clinical study by Yan et al. both total cytosolic protein content and APT-weighted CEST signal were increased in glioma compared to healthy tissue [25]. These previous studies indicate the potential clinical relevance of increased APT-weighted signal in locating active tumour tissue, an aspect that is of importance in future work to target the most aggressive area of a tumour for accurate diagnosis.

Note that, in addition to cell proliferation, it is well known that other sources of CEST signal exist that can contribute to differences in signal between tumour and healthy tissue. We find that the use of a $B_{1}$ saturation power of $3.8 \mu \mathrm{T}$ gave a stronger effect size than a $B_{1}$ of $2.5 \mu \mathrm{T}$ for separating tumour from NAWM when using $\mathrm{LD}_{\text {amide }}$. Note that, based on the theoretical optimal sensitivity of CEST MRI to amide protons when using a lower $\mathrm{B}_{1}$ saturation power $(\sim 1 \mu \mathrm{T}[10])$, this finding corroborates that there must be other sources (in part) responsible for differentiation of glioma and NAWM in our study. Based on previous studies, these other sources include the following: (i) a non-linear relationship between $\mathrm{B}_{1}$ saturation power and the ratio of $\mathrm{T}_{1}$-water relaxation times and overall water content, with the latter parameters ( $\mathrm{T}_{1}$ of water and water content) likely to be increased in the tumour compared to NAWM [25], (ii) a decrease in magnetisation transfer from semisolid macromolecules in tumour compared NAWM [26], in particular, if increased $B_{1}$ saturation power increases magnetisation transfer effects in NAWM in a stronger manner than in the tumour, (iii) contributions to the CEST signal from fast exchanging amine protons resonating around 2-3 ppm because of the relatively high $B_{1}$ saturation pulse used here [27].

Although our results indicate that differences in APTweighted CEST are found in non-enhancing glioma as well, it is thus clear that the origin of increased amideweighted CEST contrasts in non-enhancing glioma is still to be investigated. This should first be done with an extensive MR imaging protocol, including quantification of $\mathrm{T}_{1}$, high-resolution structural imaging (pre- $\&$ post-contrast $\mathrm{T}_{1}, \mathrm{~T}_{2}$-weighted FLAIR), and a CEST acquisition that allows for separation of CEST signal from amide protons, NOE and MT effects. Such a CEST acquisition could be designed to be as selective to amide protons as possible, for instance as done by Zaiss et al.[26] at ultrahigh field $(9.4 \mathrm{~T})$, with low $\mathrm{B}_{1}$ saturation power and the acquisition of a full Z-spectrum such that multi-pool Lorentzian fitting can be used to separate different CEST signal sources. To assess the extent to which amide protons are contributing to the differences in APT-weighted CEST imaging should be followed by targeted biopsies of tumour tissue for ex vivo analysis of the local environment with proteomics analysis $[25,28]$, rather than cell proliferation indices, that at minimum include measurement of cytosolic protein content and potentially further investigate specific mobile proteins and semisolid macromolecules contributing to the CEST signal.

Second, if the hyperintensities in amide-weighted CEST MRI are highlighting more aggressive tumour tissue it is important to not miss these regions when imaging nonenhancing glioma, stressing the need for having a CEST acquisition that covers the whole tumour volume. Note that although this may seem to go against the finding of Sakata et al. [2], who showed that for differentiation of low- and high-grade tumours single slice acquisition worked equally well as multi-slice acquisitions, the example of P15 in this work illustrates that hyperintense APT-weighted signal may be missed if a single slice is imaged. However, note that the work by Sakata et al. was conducted before the updated WHO tumour classification of 2016 in which the molecular diagnosis became important for grading. Moreover, advances in image acquisition for CEST MRI have enabled rapid measurement of full Z-spectra for multi-slice volumes in clinically feasible scan time, e.g. [10, 11]. Therefore, it is recommended in future work investigating CEST MRI for imaging diagnostics in non-enhancing glioma to use full tumour coverage as much as possible. This would for instance aid future research investigating whether amideweighted CEST MRI can be used to direct biopsies for accurate non-enhancing glioma diagnosis in light of the recent WHO classification or improve the use of CEST MRI for differentiation of tumour progression and radiation necrosis in treatment follow-up in glioma patients.

Note that here we found that $\mathrm{LD}_{\text {amide }}$ results in a larger effect size than MTR $_{\text {asym }}$ to differentiate non-enhancing glioma tissue from NAWM, which may be caused by finding no significant difference for $\mathrm{LD}_{\mathrm{NOE}}$ between non-enhancing glioma and NAWM. This latter finding may be as expected, as previous studies on high-grade, enhancing gliomas showing that $\mathrm{LD}_{\mathrm{NOE}}$ correlated with prognosis $[13,14]$ and grading [15], all done at $7 \mathrm{~T}$, show that stronger decreases in $\mathrm{LD}_{\mathrm{NOE}}$ compared to NAWM correlate to worse prognosis/ grade in enhancing glioma, with limited changes in $\mathrm{LD}_{\mathrm{NOE}}$ for tumours with better outcome. Moreover, at $3 \mathrm{~T}$ and in 11 high-grade glioma patients, Heo et al.[29] report only slight hypointensity in $\mathrm{LD}_{\mathrm{NOE}}$ in tumour tissue. Extrapolating these results to this study in which we only include non-enhancing 
glioma may suggest that not finding a significant $\mathrm{LD}_{\mathrm{NOE}}$ effect in the current study is plausible. Note that, as a consequence of finding limited effects of $\mathrm{LD}_{\mathrm{NOE}}$, the largest effect size in separating NAWM from non-enhancing glioma was found for $\mathrm{LD}_{\text {amide }}$ in the current study, with $\mathrm{MTR}_{\text {asym }}$ being affected by both amide- and NOE-weighted signals within the tumour and hence the noise in MTR $_{\text {asym }}$ being affected by CEST signal at both 3.5 and -3.5 ppm, illustrated by the ratio of the standard deviations compared to the group averaged values being larger for MTR $_{\text {asym }}$ (Table 2). Additionally, using MTR $_{\text {asym }}$ there was a trend to find smaller hyperintense tumour volumes than using $\mathrm{LD}_{\text {amide }}$ maps. This suggests that with using $\mathrm{MTR}_{\text {asym }}$ regions of increased amide-weighted signal within the tumour may be missed. Although we do acknowledge that these findings at this stage are specific to the set-up as used here and an increased SNR for MTR asym APT-weighted images, as can be obtained by increasing the number of acquisitions on and around $\pm 3.5 \mathrm{ppm}$ [30], can lead to increased sensitivity to areas of increased APT-weighted signal for asymmetry analysis. Future work is required to further investigate whether this finding can be confirmed for non-enhancing glioma with a set-up optimised for $\mathrm{MTR}_{\text {asym }}$ analysis.

\section{Limitations}

The number of included patients is likely too small for thorough investigation of using amide-weighted imaging for classification of non-enhancing glioma. Analyses to investigate differences between the three different tumour types, as well as IDH-mutation $(N=12)$ vs IDH-wildtype $(N=6)$ tumours were performed but resulted in no significant differences for the CEST contrasts as well as the size of the hyperintense volumes (results in Supplementary information). However, note that Paech et al. [13] and Jiang et al. [9] showed that amide proton weighted CEST signal has predictive value in assessing IDH mutation status, allowing for differentiation of low- and high-grade gliomas based on CEST imaging alone, potentially even within non-enhancing glioma solely. Future work is required to corroborate those findings.

$\mathrm{B}_{0}$-correction was carried out with the CEST data itself, rather than acquiring an external map with a low $B_{1}$ saturation power [31]. This means that the $\mathrm{B}_{0}$ correction here may be affected by hydroxyl protons, resonating close to the water peak ( 0.9 ppm [12]) causing potential inaccuracies. However, in the $\mathrm{B}_{0}$-correction performed points further than $1 \mathrm{ppm}$ away from the water peak were excluded and with visual inspection of the $\mathrm{Z}$-spectra for $\mathrm{B}_{1}$ is $2.5 \mu \mathrm{T}$ (Fig. 2) it is not likely that $\mathrm{B}_{0}$-correction is affected by widening of the peak around the $\mathrm{OH}$-peak. Note that the wider peak at the largest $\mathrm{B}_{1}$ used suggests that $\mathrm{B}_{0}$-correction for this acquisition may be contaminated by hydroxyl protons resonating near $0.9 \mathrm{ppm}$. This effectively means that the $\mathrm{B}_{0^{-}}$correction at high $B_{1}$ saturation power is overestimated and a larger shift towards the upfield frequencies is applied than strictly required. Effectively this would lead to underestimation of the APT-weighted signal at $3.5 \mathrm{ppm}$. The true extent to which this will affect the results in this work is hard to gauge. It is recommended in future work to use a separate acquisition with low $\mathrm{B}_{1}$ saturation power for $\mathrm{B}_{0}$-correction.

A steady-state CEST sequence is used in this work, as at the time of initiating this study it was deemed the most appropriate for rapid acquisition of whole tumour volume at our institute. However, there are alternatives now with long saturation blocks prior to image read-out that may result in higher signal-to-noise ratios for the CEST contrast images [11]. Future work, therefore, includes the investigation of heterogeneity in APT-weighted CEST signal in non-enhancing glioma with longer pre-saturation blocks.

\section{Conclusions}

This study illustrates that 3D pulsed CEST imaging allows for measuring heterogeneity in amide-weighted CEST signal in non-enhancing glioma. Based on the results in this study we recommend to use CEST acquisitions that cover the whole tumour volume for assessment of non-enhancing glioma, to not miss areas of hyperintense APT-weighted signal which may be small within these tumours. Future work includes investigation of the cause for increased $L_{\text {amide }}$, which is a step towards the application of APT-weighted CEST MRI for accurate diagnosis and treatment follow-up in light of the new molecular classification of non-enhancing glioma.

Supplementary Information The online version of this article (https:// doi.org/10.1007/s10334-021-00911-6) contains supplementary material, which is available to authorized users.

Author contributions Warnert: Study conception and design, Acquisition of data, Analysis and interpretation of data, Drafting of manuscript, Critical revision. Wood: Study conception and design, Analysis and interpretation of data, Drafting of manuscript, Critical revision. Incekara, Vincent, Schouten, Kros: Study conception and design, Acquisition of data, Analysis and interpretation of data, Critical revision. Barker, van den Bent: Study conception and design, Critical revision. Smits, Hernandez Tamames: Study conception and design, Analysis and interpretation of data, Critical revision.

Funding This research was conducted with support from the Dutch Cancer Society (KWF): "Non-invasive phenotypying of molecular brain tumour profiles using novel advanced MR imaging and analysis", EMCR 2015-7859, and from the Brain Tumour Charity; "Making the invisible visible: In vivo mapping of molecular biomarkers in adult diffuse glioma with CEST MRI", GN-000540. EW is funded by a "Veni Vernieuwingsimpuls" from the Dutch Association entitled "Food for thought: Oxygen delivery to the brain", Grant number 91619121. 


\section{Compliance with ethical standards}

Conflict of interest The authors declare that they have no conflict of interest.

Ethical approval All procedures performed in studies involving human participants were in accordance with the ethical standards of the institutional ethics committee of the Erasmus MC (Rotterdam, NL), which is one out of 18 accredited medical research ethics committees in the Netherlands and with the 1964 Helsinki declaration and its later amendments or comparable ethical standards.

Research involving human and animal participants This article does not contain any studies with animals performed by any of the authors.

Informed consent Informed consent was obtained from all individual participants included in the study.

Open Access This article is licensed under a Creative Commons Attribution 4.0 International License, which permits use, sharing, adaptation, distribution and reproduction in any medium or format, as long as you give appropriate credit to the original author(s) and the source, provide a link to the Creative Commons licence, and indicate if changes were made. The images or other third party material in this article are included in the article's Creative Commons licence, unless indicated otherwise in a credit line to the material. If material is not included in the article's Creative Commons licence and your intended use is not permitted by statutory regulation or exceeds the permitted use, you will need to obtain permission directly from the copyright holder. To view a copy of this licence, visit http://creativecommons.org/licenses/by/4.0/.

\section{References}

1. Choi YS, Ahn SS, Lee S-K, Chang JH, Kang S-G, Kim SH, Zhou J (2017) Amide proton transfer imaging to discriminate between low- and high-grade gliomas: added value to apparent diffusion coefficient and relative cerebral blood volume. Eur Radiol 27:3181-3189

2. Sakata A, Okada T, Yamamoto A, Kanagaki M, Fushimi Y, Okada T, Dodo T, Arakawa Y, Schmitt B, Miyamoto S, Togashi K (2015) Grading glial tumors with amide proton transfer MR imaging: different analytical approaches. J Neurooncol 122:339-348

3. Zhou J, Tryggestad E, Wen Z, Lal B, Zhou T, Grossman R, Wang S, Yan K, Fu D-X, Ford E, Tyler B, Blakeley J, Laterra J, van Zijl PCM (2011) Differentiation between glioma and radiation necrosis using molecular magnetic resonance imaging of endogenous proteins and peptides. Nat Med 17:130-134

4. Zou T, Yu H, Jiang C, Wang X, Jiang S, Rui Q, Mei Y, Zhou J, Wen $Z$ (2018) Differentiating the histologic grades of gliomas preoperatively using amide proton transfer-weighted (APTW) and intravoxel incoherent motion MRI. NMR Biomed. https:// doi.org/10.1002/nbm. 3850

5. Aum DJ, Kim DH, Beaumont TL, Leuthardt EC, Dunn GP, Kim AH (2014) Molecular and cellular heterogeneity: the hallmark of glioblastoma. Neurosurg Focus. https://doi. org/10.3171/2014.9.FOCUS14521

6. Louis DN, Perry A, Reifenberger G, von Deimling A, FigarellaBranger D, Cavenee WK, Ohgaki H, Wiestler OD, Kleihues P, Ellison DW (2016) The 2016 World Health Organization Classification of Tumors of the Central Nervous System: a summary. Acta Neuropathol 131:803-820
7. Olar A, Wani KM, Alfaro-Munoz KD, Heathcock LE, van Thuijl HF, Gilbert MR, Armstrong TS, Sulman EP, Cahill DP, VeraBolanos E, Yuan Y, Reijneveld JC, Ylstra B, Wesseling P, Aldape KD (2015) IDH mutation status and role of WHO grade and mitotic index in overall survival in grade II-III diffuse gliomas. Acta Neuropathol 129:585-596

8. Togao O, Hiwatashi A, Yamashita K, Kikuchi K, Keupp J, Yoshimoto K, Kuga D, Yoneyama M, Suzuki SO, Iwaki T, Takahashi M, Iihara K, Honda H (2017) Grading diffuse gliomas without intense contrast enhancement by amide proton transfer MR imaging: comparisons with diffusion- and perfusion-weighted imaging. Eur Radiol 27:578-588

9. Jiang S, Zou T, Eberhart CG, Villalobos MAV, Heo H-Y, Zhang Y, Wang Y, Wang X, Yu H, Du Y, van Zijl PCM, Wen Z, Zhou J (2017) Predicting IDH mutation status in grade II gliomas using amide proton transfer-weighted (APTw) MRI. Magn Reson Med 78:1100-1109

10. Jones CK, Polders D, Hua J, Zhu H, Hoogduin HJ, Zhou J, Luijten P, Van Zijl PCM (2012) In vivo three-dimensional whole-brain pulsed steady-state chemical exchange saturation transfer at $7 \mathrm{~T}$. Magn Reson Med 67:1579-1589

11. Deshmane A, Zaiss M, Lindig T, Herz K, Schuppert M, Gandhi C, Bender B, Ernemann U, Scheffler K (2019) 3D gradient echo snapshot CEST MRI with low power saturation for human studies at 3T. Magn Reson Med 81:2412-2423

12. van Zijl PCM, Lam WW, Xu J, Knutsson L, Stanisz GJ (2018) Magnetization transfer contrast and chemical exchange saturation transfer MRI. Features and analysis of the field-dependent saturation spectrum. Neuroimage 168:222-241

13. Paech D, Windschuh J, Oberhollenzer J, Dreher C, Sahm F, Meissner JE, Goerke S, Schuenke P, Zaiss M, Regnery S, Bickelhaupt S, Bäumer P, Bendszus M, Wick W, Unterberg A, Bachert P, Ladd ME, Schlemmer HP, Radbruch A (2018) Assessing the predictability of IDH mutation and MGMT methylation status in glioma patients using relaxation-compensated multipool CEST MRI at 7.0 T. Neuro Oncol 20:1661-1671

14. Regnery S, Adeberg S, Dreher C, Oberhollenzer J, Meissner JE, Goerke S, Windschuh J, Deike-Hofmann K, Bickelhaupt S, Zaiss M, Radbruch A, Bendszus M, Wick W, Unterberg A, Rieken S, Debus J, Bachert P, Ladd M, Schlemmer HP, Paech D (2018) Chemical exchange saturation transfer MRI serves as predictor of early progression in glioblastoma patients. Oncotarget 9:28772-28783

15. Heo HY, Jones CK, Hua J, Yadav N, Agarwal S, Zhou J, van Zijl PCM, Pillai JJ (2016) Whole-brain amide proton transfer (APT) and nuclear overhauser enhancement (NOE) imaging in glioma patients using low-power steady-state pulsed chemical exchange saturation transfer (CEST) imaging at 7T. J Magn Reson Imaging $44: 41-50$

16. Block W, Pauly J, Kerr A, Nishimura D (1997) Consistent fat suppression with compensated spectral-spatial pulses. Magn Reson Med 38:198-206

17. Zhou J (2011) Amide proton transfer imaging of the human brain. Methods Mol Biol 711:227-237

18. Scheidegger R, Wong ET, Alsop DC (2014) Contributors to contrast between glioma and brain tissue in chemical exchange saturation transfer sensitive imaging at 3 Tesla. Neuroimage 99:256-268

19. Jones CK, Huang A, Xu J, Edden RAE, Schär M, Hua J, Oskolkov N, Zacà D, Zhou J, McMahon MT, Pillai JJ, van Zijl PCM (2013) Nuclear Overhauser enhancement (NOE) imaging in the human brain at 7T. Neuroimage 77:114-124

20. Kuroda J, Kinoshita M, Tanaka H, Nishida T, Nakamura H, Watanabe Y, Tomiyama N, Fujinaka T, Yoshimine T (2012) Cardiac cycle-related volume change in unruptured cerebral aneurysms: a detailed volume quantification study using 4-dimensional CT angiography. Stroke 43:61-66 
21. Windschuh J, Zaiss M, Meissner JE, Paech D, Radbruch A, Ladd ME, Bachert P (2015) Correction of B1-inhomogeneities for relaxation-compensated CEST imaging at 7T. NMR Biomed 28:529-537

22. Yushkevich PA, Piven J, Hazlett HC, Smith RG, Ho S, Gee JC, Gerig G (2006) User-guided 3D active contour segmentation of anatomical structures: Significantly improved efficiency and reliability. Neuroimage 31:1116-1128

23. Jiang S, Eberhart CG, Zhang Y, Heo H-Y, Wen Z, Blair L, Qin H, Lim M, Quinones-Hinojosa A, Weingart JD, Barker PB, Pomper MG, Laterra J, van Zij1 PCM, Blakeley JO, Zhou J (2017) Amide proton transfer-weighted magnetic resonance image-guided stereotactic biopsy in patients with newly diagnosed gliomas. Eur J Cancer 83:9-18

24. Jiang S, Eberhart CG, Lim M, Heo H-Y, Zhang Y, Blair L, Wen Z, Holdhoff M, Lin D, Huang P, Qin H, Quinones-Hinojosa A, Weingart JD, Barker PB, Pomper MG, Laterra J, van Zijl PCM, Blakeley JO, Zhou J (2019) Identifying recurrent malignant glioma after treatment using amide proton transfer-weighted MR imaging: a validation study with image-guided stereotactic biopsy. Clin cancer Res an Off J Am Assoc Cancer Res 25:552-561

25. Yan K, Fu Z, Yang C, Zhang K, Jiang S, Lee DH, Heo HY, Zhang Y, Cole RN, Van Eyk JE, Zhou J (2015) Assessing amide proton transfer (APT) MRI contrast origins in $9 \mathrm{~L}$ gliosarcoma in the rat brain using proteomic analysis. Mol Imaging Biol 17:479-487

26. Zaiss M, Schuppert M, Deshmane A, Herz K, Ehses P, Füllbier L, Lindig T, Bender B, Ernemann U, Scheffler K (2018) Chemical exchange saturation transfer MRI contrast in the human brain at 9.4 T. Neuroimage 179:144-155
27. Harris RJ, Cloughesy TF, Liau LM, Prins RM, Antonios JP, Li D, Yong WH, Pope WB, Lai A, Nghiemphu PL, Ellingson BM (2015) PH-weighted molecular imaging of gliomas using amine chemical exchange saturation transfer MRI. Neuro Oncol 17:1514-1524

28. Xu J, Zaiss M, Zu Z, Li H, Xie J, Gochberg DF, Bachert P, Gore JC (2014) On the origins of chemical exchange saturation transfer (CEST) contrast in tumors at 9.4T. NMR Biomed 27:406-416

29. Heo HY, Zhang Y, Lee DH, Hong X, Zhou J (2016) Quantitative assessment of amide proton transfer (APT) and nuclear overhauser enhancement (NOE) imaging with extrapolated semi-solid magnetization transfer reference (EMR) signals: application to a rat glioma model at 4.7 tesla. Magn Reson Med 75:137-149

30. Zhou J, Heo HY, Knutsson L, van Zijl PCM, Jiang S (2019) APTweighted MRI: Techniques, current neuro applications, and challenging issues. J Magn Reson Imaging. https://doi.org/10.1002/ jmri.26645

31. Kim M, Gillen J, Landman BA, Zhou J, Van Zijl PCM (2009) Water saturation shift referencing (WASSR) for chemical exchange saturation transfer (CEST) experiments. Magn Reson Med. https://doi.org/10.1002/mrm.21873

Publisher's Note Springer Nature remains neutral with regard to jurisdictional claims in published maps and institutional affiliations. 